\title{
Modeling nonlinear ultrasound propagation in heterogeneous media with power law absorption using a $k$-space pseudospectral method
}

\author{
Bradley E. Treeby ${ }^{\mathrm{a})}$ \\ Research School of Engineering, College of Engineering and Computer Science, The Australian National \\ University, Canberra ACT 0200, Australia \\ Jiri Jaros and Alistair P. Rendell \\ Research School of Computer Science, College of Engineering and Computer Science, The Australian \\ National University, Canberra ACT 0200, Australia \\ B. T. Cox \\ Department of Medical Physics and Bioengineering, University College London, Gower Street, \\ London WC1E 6BT, United Kingdom
}

(Received 6 September 2011; revised 3 April 2012; accepted 11 April 2012)

\begin{abstract}
The simulation of nonlinear ultrasound propagation through tissue realistic media has a wide range of practical applications. However, this is a computationally difficult problem due to the large size of the computational domain compared to the acoustic wavelength. Here, the $k$-space pseudospectral method is used to reduce the number of grid points required per wavelength for accurate simulations. The model is based on coupled first-order acoustic equations valid for nonlinear wave propagation in heterogeneous media with power law absorption. These are derived from the equations of fluid mechanics and include a pressure-density relation that incorporates the effects of nonlinearity, power law absorption, and medium heterogeneities. The additional terms accounting for convective nonlinearity and power law absorption are expressed as spatial gradients making them efficient to numerically encode. The governing equations are then discretized using a $k$-space pseudospectral technique in which the spatial gradients are computed using the Fourier-collocation method. This increases the accuracy of the gradient calculation and thus relaxes the requirement for dense computational grids compared to conventional finite difference methods. The accuracy and utility of the developed model is demonstrated via several numerical experiments, including the 3D simulation of the beam pattern from a clinical ultrasound probe.
\end{abstract}

(C) 2012 Acoustical Society of America. [http://dx.doi.org/10.1121/1.4712021]

PACS number(s): 43.25.Jh, 43.20.Bi, 43.35.Bf, 43.25.Cb [TDM] Pages: 4324-4336

\section{INTRODUCTION}

The simulation of ultrasound propagation through soft biological tissue has a wide range of practical applications. These include the design of transducers for diagnostic and therapeutic ultrasound, the development of new signal processing and imaging techniques, studying the aberration of ultrasound beams in heterogeneous media, ultrasonic tissue classification, training ultrasonographers to use ultrasound equipment and interpret ultrasound images, model-based medical image registration, and treatment planning and dosimetry for high-intensity focused ultrasound. ${ }^{1}$ The most general approach for ultrasound simulation is to directly solve the equations of continuum mechanics. However, this is a computationally difficult problem due to the large size of the region of interest in relation to the size of the acoustic wavelength. For example, a typical diagnostic ultrasound image formed using a $3 \mathrm{MHz}$ curvilinear transducer has a depth penetration of around $15 \mathrm{~cm}$. This distance is on the order of 300 acoustic wavelengths at the fundamental fre-

\footnotetext{
${ }^{\text {a) }}$ Author to whom correspondence should be addressed. Electronic mail: bradley.treeby@anu.edu.au
}

quency, and 600 wavelengths at the second harmonic. Established numerical methods such as the finite difference or finite element methods require on the order of 10 grid points per wavelength to achieve acceptable accuracy. This equates to a computational domain with thousands of grid points in each spatial dimension. Consequently, many simulations of interest are simply intractable, or require very large amounts of computer memory and can take days or weeks to run. ${ }^{2}$

To reduce this computational burden, simplifying assumptions are frequently made. For modeling the beam patterns from ultrasound transducers, a common approach is to only consider one-way (or forward) wave propagation (see Huijssen and Verweij ${ }^{3}$ for a recent review). If the problem is axisymmetric, the governing equations can also be solved in $2 \mathrm{D} .{ }^{4}$ However, these approaches are unable to account for all aspects of nonlinear wave propagation in heterogeneous media. For the simulation of diagnostic ultrasound images, a Green's function method is also often used. ${ }^{5}$ In this case, the scattering medium is modeled as series of point sources in a homogeneous background (the widely used FIELD II program is based on this approach). However, this does not account for more complex acoustic phenomena, for example, multiple scattering or nonlinearity. Given the 
wide range of possible applications, there is a strong motivation for the development of new ultrasound simulation tools with less restrictive assumptions and improved computational efficiency.

Here, a computationally efficient approach for the simulation of nonlinear wave propagation is derived using a $k$-space pseudospectral method. ${ }^{6}$ In Sec. II, existing methods for modeling ultrasound propagation in tissue realistic media are reviewed. In this context, approaches for modeling both heterogeneous media and power law absorption are discussed. In Sec. III, governing equations suitable for modeling nonlinear ultrasound propagation in heterogeneous media with power law absorption are then developed. In Sec. IV, these equations are discretized using a $k$-space pseudospectral method. The spectral calculation of the spatial derivatives is performed using the Fourier-collocation method and significantly relaxes the requirement for dense computational grids compared to finite difference methods. In Sec. V, several numerical experiments are presented to illustrate the accuracy and efficacy of the developed model. Discussion and summary are then given in Sec. VI, with further details of the computer code provided in the Appendix.

\section{TISSUE REALISTIC ULTRASOUND MODELING}

\section{A. Model requirements}

The development of accurate models for ultrasound propagation in soft tissue requires the consideration of three important factors. (1) In most cases the amplitude of the acoustic waves is sufficiently large that the wave propagation is nonlinear. For example, modern ultrasound scanners rely on nonlinear wave propagation for tissue harmonic imaging which gives images with improved clarity and contrast. (2) The material properties of biological tissue (e.g., the sound speed and density) are weakly heterogeneous, with variations between the different soft tissue types and water on the order of 5\%. ${ }^{1}$ (3) The tissue is absorbing at ultrasonic frequencies with the absorption following a frequency power law. In the context of nonlinear wave propagation, an accurate model of acoustic absorption is of particular importance as the generation of higher frequency harmonics via nonlinearity is delicately balanced with their absorption.

When considered individually as extensions to the standard equations of linear acoustics, each of these factors has been extensively studied. It is the unification of these effects into a consistent set of coupled first-order equations, in addition to the efficient solution of these equations, that is the subject of interest here. The use of first-order governing equations rather than the corresponding second-order wave equation has several advantages. First, it allows the pressure and particle velocity to be computed on staggered grids which improves accuracy. Second, it allows the use of an anisotropic perfectly matched layer (PML) for absorbing the acoustic waves at the edges of the computational domain. ${ }^{6}$ Third, it provides an intuitive way to directly include both mass and force sources in the discrete equations. Finally, the explicit calculation of the particle velocity allows the vector components of acoustic intensity to be directly computed.
This is of particular relevance when modeling the heat deposition from therapeutic ultrasound probes.

\section{B. Accounting for tissue heterogeneities}

Over the last half a century, a large number of researchers have contributed to an extensive body of knowledge on the nonlinear propagation of acoustic waves. ${ }^{7}$ From a theoretical perspective, the required governing equations can be derived by including second order (and in some cases third order) terms in the conservation equations and pressuredensity relation. However, despite the long history of nonlinear acoustics, most rigorous derivations are based on the assumption of a homogeneous medium with thermoviscous absorption. In particular, there have been very few attempts to consider acoustic heterogeneities at the level of the governing equations. (Two recent exceptions are the papers by Taraldsen, ${ }^{8}$ who derived a heterogeneous Westervelt equation in Lagrangian coordinates, and Coulouvrat, ${ }^{9}$ who considered the case of a heterogeneous and moving turbulent fluid.) While it is straightforward to consider heterogeneous medium parameters in the conservation equations, the derivation of a pressure-density relation valid for nonlinear wave propagation in heterogeneous media is more difficult to find in the literature.

The most common approach to modeling heterogeneous medium parameters is to assume that the effects of nonlinearity and heterogeneity are sufficiently small that their interactions can be neglected. In this way, an appropriate wave equation can be formed by combining the additional terms from the linear wave equation for heterogeneous media with those from the nonlinear wave equation for homogeneous media. For example, Hallaj et al., ${ }^{10}$ and Pinton et al., ${ }^{2}$ both utilized a Westervelt equation augmented with the heterogeneous density term from the linear wave equation. Jing and Cleveland ${ }^{11}$ presented a similar wave equation including local nonlinear effects. This was then reduced to a Khokhlov-Zabolotskaya-Kuznetsov (KZK) equation suitable for heterogeneous media. Comparisons of simulations using this equation with experimental measurements of ultrasound propagation through a heterogeneous gel layer showed good agreement. An analogous approach was taken by Verweij and Huijssen ${ }^{12}$ and Jing and Clement ${ }^{13}$ where both the nonlinearity and heterogeneity terms were introduced as contrast source terms. Similarly, Averyanov et al., supplemented a linear parabolic wave equation for heterogeneous media with additional terms describing the effects of nonlinearity and absorption. ${ }^{14}$ While the accuracy of these wave equations for modeling nonlinear wave propagation in weakly heterogeneous media is well established, they do not provide heterogeneous forms of the conservation and pressure-density equations which can be solved as a set of coupled first-order equations.

\section{Accounting for power law acoustic absorption}

Classical lossy wave equations based on the inclusion of viscosity and thermal conduction into the governing equations yield an acoustic absorption term that is proportional to frequency squared. However, the absorption mechanisms in 
soft biological tissue are significantly more complex (including vibrational, structural, and chemical relaxations) which leads to an experimentally observed attenuation of the form

$$
\alpha=\alpha_{0} \omega^{y}
$$

where the power law exponent $y$ is typically in the range $1-1.5$. $^{1}$ To account for this difference, the thermoviscous absorption term can be replaced with an alternate loss term. This idea was first proposed by Blackstock who replaced the thermoviscous term in the lossy Burgers equation with a general absorption operator. ${ }^{15}$ Szabo later derived a causal form of this operator to account for power law absorption with an arbitrary frequency dependence. ${ }^{16,17}$ This was derived in the form of a time domain convolution operator and was used to replace the classical thermoviscous absorption terms in the KZK, Burgers, and Westervelt equations. ${ }^{16}$ Similar convolution operators for the KZK equation ${ }^{18}$ and Kuznetsov's equation ${ }^{19}$ have also been derived.

Although Szabo's lossy operator correctly incorporates the required power law behavior, the operator is dependent on the time history of the pressure field which makes it difficult to encode in a memory efficient manner. As an alternative, Chen and Holm $^{20}$ derived a lossy operator based on the fractional Laplacian. This was similarly used to replace the absorption terms in the KZK, Burgers, and Westervelt equations. ${ }^{21}$ This operator was later extended to correctly account for power law dispersion as required by the Kramers-Kronig relations. $^{22}$ In comparison to Szabo's operator, the computation of the fractional Laplacian only depends on the values of the pressure field at the current time. This makes the operator efficient to compute, particularly using Fourier-based pseudospectral and $k$-space methods. ${ }^{22,23}$

An alternative approach to using a phenomenological operator to account for power law absorption is to explicitly consider the absorption as a sum of relaxation processes. This is based on a physical analogy with the different absorption mechanisms in tissue which act as relaxation processes with varying relaxation times. Models for both a continuous distribution of relaxation parameters ${ }^{24}$ and a discrete set of relaxation parameters ${ }^{25}$ have been proposed. However, despite the physical appeal of such models, for biological materials the individual relaxation processes and their relaxation times are not generally known. Consequently, the model parameters must instead be derived using a fitting procedure based on experimental data. In this case, the derived relaxation parameters do not necessarily have any direct connection with the physical absorption mechanisms and thus can also be considered as phenomenological terms. The discrete relaxation model has been applied to the linear, ${ }^{25} \mathrm{KZK},{ }^{26}$ and Westervelt ${ }^{2}$ equations. For the latter, two relaxation processes were found to be sufficient to approximate power law absorption over a $12 \mathrm{MHz}$ frequency range. ${ }^{2}$

Given that only a small number of relaxation parameters are generally needed to approximate power law absorption over a given frequency range, there is not a clear argument for using a phenomenological absorption operator over a relaxation operator, or vice-versa. Indeed, Näsholm and Holm have recently shown that, under certain conditions, fractional loss operators can be derived from a continuum of relaxation processes, rendering the two approaches equivalent. ${ }^{27}$ On one hand, the relaxation approach is more general and is able to model absorption with an arbitrary frequency dependence. On the other hand, the extraction of the relaxation parameters needed for the model requires an a priori fitting procedure for each value of absorption and range of frequencies under consideration. If the objective is specifically to model power law absorption (as is the case here), it is easier to directly use an operator that can account for this behavior.

\section{NONLINEAR GOVERNING EQUATIONS FOR HETEROGENEOUS MEDIA}

\section{A. General nonlinear equations}

The equations required to describe the nonlinear propagation of compressional acoustic waves through heterogeneous fluid media can be obtained directly from the equations of fluid mechanics. Under the assumption of a quiescent, isotropic, and inviscid medium (acoustic absorption is explicitly considered later as an energy loss term), the momentum and mass conservation equations can, respectively, be written in a Eulerian coordinate system as ${ }^{7}$

$$
\begin{aligned}
& \rho_{0} \frac{\partial \mathbf{u}}{\partial t}+\nabla p=-\rho \frac{\partial \mathbf{u}}{\partial t}-\frac{1}{2} \rho_{0} \nabla\left(\mathbf{u}^{2}\right), \\
& \frac{\partial \rho}{\partial t}+\nabla \cdot\left(\rho_{0} \mathbf{u}\right)=-\nabla \cdot(\rho \mathbf{u}) .
\end{aligned}
$$

Here $p$ and $\rho$ are the acoustic pressure and density, $\mathbf{u}$ is the acoustic particle velocity where $\mathbf{u}^{2}=\mathbf{u} \cdot \mathbf{u}$, and $\rho_{0}$ is the ambient density. Note, in these and subsequent expressions, only terms up to second order in the acoustic variables are retained. This is sufficient for modeling the finite amplitude effects of interest here.

A nonlinear pressure-density relation for an arbitrary fluid medium can then be obtained by expanding the state equation $\hat{p}=\hat{p}(\hat{\rho}, \hat{s})$ in a Taylor series about the ambient density and entropy. Here the ${ }^{\wedge}$ symbol is used to denote a total quantity, i.e., the sum of ambient and acoustic parts. Following Lighthill, ${ }^{28}$ it is assumed that the effects of nonlinearity and changes in entropy (which are due to acoustic absorption) are both second order. Consequently, higher order entropy terms can be discarded. Considering the change in the total pressure of a fluid element for a small but finite time step $\delta t=t_{1}-t_{0}$, the Taylor series expansion can then be written as

$$
\begin{aligned}
\hat{p}\left(t_{1}\right)-\hat{p}\left(t_{0}\right)= & \left(\frac{\partial \hat{p}}{\partial \hat{\rho}}\right)_{\hat{s}}\left(\hat{\rho}\left(t_{1}\right)-\hat{\rho}\left(t_{0}\right)\right)+\frac{1}{2}\left(\frac{\partial^{2} \hat{p}}{\partial \hat{\rho}^{2}}\right)_{\hat{s}} \\
& \times\left(\hat{\rho}\left(t_{1}\right)-\hat{\rho}\left(t_{0}\right)\right)^{2}+\left(\frac{\partial \hat{p}}{\partial \hat{s}}\right)_{\hat{\rho}}\left(\hat{s}\left(t_{1}\right)-\hat{s}\left(t_{0}\right)\right) .
\end{aligned}
$$

If the ambient medium parameters are heterogeneous, the change in the total density $\hat{\rho}\left(t_{1}\right)-\hat{\rho}\left(t_{0}\right)$ can arise either due to local acoustic perturbations, or due to the displacement of 
the fluid element under consideration to a new position in which the ambient density is different. ${ }^{29}$ If $\hat{\rho}$ is written as a function of both position $x$ and time $t$, the change in $\hat{\rho}$ between $t_{0}$ and $t_{1}$ can similarly be expanded using a Taylor series. This yields the expression

$$
\hat{\rho}\left(t_{1}\right)-\hat{\rho}\left(t_{0}\right)=\left(\frac{\partial \hat{\rho}}{\partial t}\right)_{x}\left(t_{1}-t_{0}\right)+\left(\frac{\partial \hat{\rho}}{\partial x}\right)_{t}\left(\zeta_{1}-\zeta_{0}\right),
$$

where $\zeta_{1}$ and $\zeta_{0}$ are the positions of the fluid element at $t_{1}$ and $t_{0}$. The first term is the conventional Eulerian description of the acoustic density $\rho$ (assuming the medium is quiescent), while the second term is due to the displacement of the fluid element. This is equivalent to describing the change in density within a fixed element in a Eulerian coordinate system which has an additional component due to the displacement of the surrounding fluid. Defining the particle displacement vector as $\mathbf{d}=\zeta_{1}-\zeta_{0}$ and writing the spatial derivative of $\hat{\rho}$ at constant time in vector notation as

$$
\left(\frac{\partial \hat{\rho}}{\partial x}\right)_{t} \equiv \nabla \hat{\rho}\left(t_{0}\right)=\nabla \rho_{0}
$$

Eq. (4) then becomes

$$
\hat{\rho}\left(t_{1}\right)-\hat{\rho}\left(t_{0}\right)=\rho+\mathbf{d} \cdot \nabla \rho_{0} .
$$

Assuming the medium is initially in thermodynamic equilibrium, the equivalent spatial gradients of $\hat{s}$ and $\hat{p}$ are zero giving $\hat{s}\left(t_{1}\right)-\hat{s}\left(t_{0}\right)=s$ and $\hat{p}\left(t_{1}\right)-\hat{p}\left(t_{0}\right)=p$. Using these expressions and making the substitutions ${ }^{7}$

$$
A \equiv \rho_{0}\left(\frac{\partial \hat{p}}{\partial \hat{\rho}}\right)_{\hat{s}}=\rho_{0} c_{0}^{2}, \quad B \equiv \rho_{0}^{2}\left(\frac{\partial^{2} \hat{p}}{\partial \hat{\rho}^{2}}\right)_{\hat{s}}
$$

(where the first equation above defines the isentropic sound speed $c_{0}$ ), Eq. (3) can then be written in the form

$$
\begin{aligned}
p= & c_{0}^{2}\left(\rho+\mathbf{d} \cdot \nabla \rho_{0}\right)+\left(\frac{\partial \hat{p}}{\partial \hat{s}}\right)_{\hat{\rho}} s \\
& +\frac{B}{2 A} \frac{c_{0}^{2}}{\rho_{0}}\left(\rho^{2}+\left(\mathbf{d} \cdot \nabla \rho_{0}\right)^{2}+2 \rho \mathbf{d} \cdot \nabla \rho_{0}\right) .
\end{aligned}
$$

Here $B / A$ is the parameter of nonlinearity which characterizes the relative contribution of finite-amplitude effects to the sound speed. ${ }^{7}$ Note, in the linear case, an equivalent relation can be derived by considering the Lagrangian derivative of the state equation. ${ }^{30}$

The remaining entropy term in Eq. (6) represents an energy loss or acoustic absorption term. In the case of thermoviscous media, this can be related to the thermal conductivity and specific heat capacity of the medium by explicit consideration of the appropriate energy conservation equation. ${ }^{7}$ More generally (by analogy with Blackstock ${ }^{15}$ ), this can be replaced by a phenomenological loss term of form

$$
\left(\frac{\partial \hat{p}}{\partial \hat{s}}\right)_{\hat{\rho}} s=-\left(\frac{\partial \hat{p}}{\partial \hat{\rho}}\right)_{\hat{s}}\left(\frac{\partial \hat{\rho}}{\partial \hat{s}}\right)_{\hat{p}} s \equiv-c_{0}^{2} L \rho,
$$

where $L$ is a general loss operator. For modeling power law absorption, it is convenient to define $L$ as a derivative operator based on the fractional Laplacian ${ }^{22}$

$$
L=\tau \frac{\partial}{\partial t}\left(-\nabla^{2}\right)^{y / 2-1}+\eta\left(-\nabla^{2}\right)^{(y+1) / 2-1} .
$$

Here $\tau$ and $\eta$ are absorption and dispersion proportionality coefficients given by $\tau=-2 \alpha_{0} c_{0}^{y-1}$ and $\eta=2 \alpha_{0} c_{0}^{y} \tan (\pi y / 2)$, $\alpha_{0}$ is the power law prefactor in $\mathrm{Np}(\mathrm{rad} / \mathrm{s})^{-y} \mathrm{~m}^{-1}$, and $y$ is the power law exponent. The two terms in $L$ separately account for power law absorption and dispersion for $0<y<3$ and $y \neq 1$ under particular smallness conditions. ${ }^{22}$ These conditions are generally satisfied for the range of attenuation parameters observed in soft biological tissue (for very high values of absorption and frequency the accuracy of the loss operator decreases due to second-order effects ${ }^{23}$ ). The use of a fractional derivative in the pressure-density relation can also be related to a general relaxation relationship between the temperature gradient and resulting heat flux which leads to a fractional entropy equation. ${ }^{31}$

\section{B. Reduced nonlinear equations}

While the general first-order equations derived in the previous section could be directly solved using standard numerical techniques, it is both unwieldy and unnecessary to do so. For many applications in biomedical ultrasonics, it is sufficient to consider only cumulative nonlinear effects. ${ }^{32}$ We also make the assumption that the effect of acoustic heterogeneities on the wave field can be considered as secondorder. Any higher order heterogeneity terms or interactions between nonlinearity and heterogeneity terms can then also be discarded.

Returning to the momentum and mass conservation equations given in Eq. (2), following the approach taken by Aanonsen et al., ${ }^{7,33}$ the second-order terms which appear on the right hand side can now be re-written in terms of the acoustic Lagrangian density via the repeated substitution of the homogeneous acoustic equations in linearized form (using the premise that the substitution of first-order equations into second-order terms yields third-order errors). This gives the expressions

$$
\begin{aligned}
& \rho_{0} \frac{\partial \mathbf{u}}{\partial t}+\nabla p=-\nabla \mathcal{L}, \\
& \frac{\partial \rho}{\partial t}+\nabla \cdot\left(\rho_{0} \mathbf{u}\right)=\frac{1}{c_{0}^{2}} \frac{\partial \mathcal{L}}{\partial t}+\frac{1}{\rho_{0} c_{0}^{4}} \frac{\partial p^{2}}{\partial t},
\end{aligned}
$$

where $\mathcal{L}$ is the second-order Lagrangian density given by

$$
\mathcal{L}=\frac{1}{2} \rho_{0} \mathrm{u}^{2}-\frac{p^{2}}{2 \rho_{0} c_{0}^{2}}
$$

This characterizes the difference between the kinetic and potential energy density of the acoustic wave. If only cumulative nonlinear effects are important, the Lagrangian density can be set to zero which leaves

Treeby et al.: Modeling nonlinear ultrasound propagation

4327 


$$
\begin{aligned}
& \frac{\partial \mathbf{u}}{\partial t}+\frac{1}{\rho_{0}} \nabla p=0, \\
& \frac{\partial \rho}{\partial t}+\nabla \cdot\left(\rho_{0} \mathbf{u}\right)=\frac{1}{\rho_{0} c_{0}^{4}} \frac{\partial p^{2}}{\partial t} .
\end{aligned}
$$

If the governing equations are solved using spectral methods (as is the case here), it is convenient to re-write the convective nonlinear term that appears in the mass conservation equation given in Eq. (9b) in its original form as a spatial gradient. This is because spatial gradients can be computed spectrally, while temporal gradients require the use of a finite difference approximation as well as additional storage. By following the series of substitutions that yield Eq. (8b) from Eq. (2b), it can be shown that the final term is equivalent to the expression $-2 \rho \nabla \cdot \mathbf{u}$ (to second order). Using this substitution gives an alternate form of the mass conservation equation valid for modeling cumulative nonlinear effects. Combined with the momentum conservation equation and the pressure-density relation, the full set of coupled equations can now be written as

$$
\begin{aligned}
& \frac{\partial \mathbf{u}}{\partial t}=-\frac{1}{\rho_{0}} \nabla p, \\
& \frac{\partial \rho}{\partial t}=-\left(2 \rho+\rho_{0}\right) \nabla \cdot \mathbf{u}-\mathbf{u} \cdot \nabla \rho_{0}, \\
& p=c_{0}^{2}\left(\rho+\mathbf{d} \cdot \nabla \rho_{0}+\frac{B}{2 A} \frac{\rho^{2}}{\rho_{0}}-L \rho\right),
\end{aligned}
$$

where the loss operator $L$ is defined in Eq. (7). The momentum conservation equation is identical to the linear case, ${ }^{34}$ while the mass conservation equation has an additional term which accounts for a convective nonlinearity in which the particle velocity contributes to the wave velocity. The four terms within the pressure-density relation separately account for linear wave propagation, heterogeneities in the ambient density, material nonlinearity, and power law absorption and dispersion (the sound speed $c_{0}$ can also be heterogeneous). Note, the $\mathbf{u} \cdot \nabla \rho_{0}$ term in the mass conservation equation and the d. $\nabla \rho_{0}$ term in the pressure-density relation cancel when these equations are combined (or solved as coupled equations). Consequently, these terms are not included in the discrete equations given in the following section to improve computational efficiency. ${ }^{35}$

Using the mass conservation equation in the form given in Eq. (9b) and neglecting higher order absorption terms, the coupled governing equations can also be combined to give a modified form of the Westervelt equation valid for heterogeneous media with power law absorption

$$
\nabla^{2} p-\frac{1}{c_{0}^{2}} \frac{\partial^{2} p}{\partial t^{2}}-\frac{1}{\rho_{0}} \nabla \rho_{0} \cdot \nabla p+\frac{\beta}{\rho_{0} c_{0}^{4}} \frac{\partial^{2} p^{2}}{\partial t^{2}}-L \nabla^{2} p=0,
$$

where $\beta=1+B / 2 A$ is the coefficient of nonlinearity (an equivalent expression assimilating the Laplacian into the loss operator is given in Ref. 36). This expression can be considered as a particular case of the generalized Westervelt equation discussed by Taraldsen. ${ }^{8}$

\section{NUMERICAL IMPLEMENTATION}

\section{A. Overview of the $k$-space method}

Closely connected with the development of accurate governing equations for describing ultrasound propagation in tissue is the issue of their efficient solution. In a standard finite difference method, spatial gradients are computed locally based on the function values at neighboring grid points. As an alternative, it is also possible to calculate spatial gradients globally using the function values across the whole domain via spectral methods. This increases the accuracy of the gradient calculation and thus reduces the number of grid points required per wavelength for a given level of accuracy. For smoothly varying fields, spatial gradients can be calculated with spectral accuracy up to the Nyquist limit (two grid points per wavelength). Often the spectral calculation of spatial gradients is combined with the finite difference calculation of temporal gradients. However, the finite difference approximation introduces unwanted numerical dispersion into the solution that can only be controlled by reducing the size of the time step or increasing the order of the approximation.

Fortunately, for the standard linear wave equation valid for homogeneous and lossless media, an exact finite difference scheme for the temporal derivative exists. This can be used to derive an exact pseudospectral discretization of both the second-order wave equation, ${ }^{37}$ and the corresponding coupled first-order conservation equations and pressuredensity relation. ${ }^{6}$ This approach is known as the $k$-space pseudospectral method (or simply the $k$-space method), because the difference between the exact and standard finite difference approximations reduces to an operator in the spatial frequency domain (referred to herein as the $k$-space operator). In the case of heterogeneous and absorbing media, the temporal discretization is no longer exact. However, if these perturbations are small, the $k$-space operator still reduces the unwanted numerical dispersion associated with the finite difference approximation of the time derivative (see discussion in Sec. V A). ${ }^{6,37}$ Recently, Jing and Clement ${ }^{13}$ presented a $k$ space method based on the Westervelt equation for thermoviscous media in which the absorption and nonlinearity terms were introduced as contrast source terms. Here, the $k$ space pseudospectral method described by Tabei et al., ${ }^{6}$ is used to discretize the coupled governing equations derived in Sec. III B.

\section{B. Discrete $\boldsymbol{k}$-space equations}

Solving for the particle velocity in Eq. (10a) using an explicit first-order forward difference and for the acoustic density in Eq. (10b) using an implicit first-order forward difference, the conservation equations written in discrete form using the Fourier-collocation $k$-space pseudospectral method are given by 


$$
\begin{aligned}
& \frac{\partial}{\partial \xi} p^{n}=\mathbb{F}^{-1}\left\{i k_{\xi} \kappa \mathbb{F}\left\{p^{n}\right\}\right\}, \\
& u_{\xi}^{n+1}=u_{\xi}^{n}-\frac{\Delta t}{\rho_{0}} \frac{\partial}{\partial \xi} p^{n}, \\
& \frac{\partial}{\partial \xi} u_{\xi}^{n+1}=\mathbb{F}^{-1}\left\{i k_{\xi} \kappa \mathbb{F}\left\{u_{\xi}^{n+1}\right\}\right\}, \\
& \rho_{\xi}^{n+1}=\frac{\rho_{\xi}^{n}-\Delta t \rho_{0} \frac{\partial}{\partial \xi} u_{\xi}^{n+1}}{1+2 \Delta t \frac{\partial}{\partial \xi} u_{\xi}^{n+1}} .
\end{aligned}
$$

The acoustic density is artificially divided into Cartesian components to allow an anisotropic PML to be applied. ${ }^{38}$ Here, $\mathbb{F}$ and $\mathbb{F}^{-1}$ denote the forward and inverse spatial Fourier transform, the superscript $n$ and $n+1$ denote the function values at current and next time points, respectively, $i$ is the imaginary unit, $k_{\xi}$ is the wavenumber in the $\xi$ direction, $\Delta t$ is the time step, and $\kappa$ is the $k$-space operator given by ${ }^{6,39}$

$$
\kappa=\operatorname{sinc}\left(c_{\text {ref }} k \Delta t / 2\right),
$$

where $k^{2}=\sum_{\xi} k_{\xi}^{2}$, and $c_{\text {ref }}$ is a reference sound speed (see discussion in Sec. V A). Equations (11a)-(11d) are repeated for each Cartesian direction in $\mathbb{R}^{N}$ where $\xi=x$ in $\mathbb{R}^{1}$, $\xi=x, y$ in $\mathbb{R}^{2}$, and $\xi=x, y, z$ in $\mathbb{R}^{3}$. Using the Fourier transform of the negative fractional Laplacian ${ }^{20}$

$$
\mathbb{F}\left\{\left(-\nabla^{2}\right)^{a} \rho\right\}=k^{2 a} \mathbb{F}\{\rho\},
$$

the corresponding pressure-density relation in discrete form can be written as

$$
p^{n+1}=c_{0}^{2}\left(\rho^{n+1}+\frac{B}{2 A} \frac{1}{\rho_{0}}\left(\rho^{n+1}\right)^{2}-L_{d}\right) \text {, }
$$

where the total acoustic density is given by $\rho=\sum_{\xi} \rho_{\xi}$ and the discrete loss term is

$$
\begin{aligned}
L_{d}= & -\tau \mathbb{F}^{-1}\left\{k^{y-2} \mathbb{F}\left\{\rho_{0} \sum_{\xi} \frac{\partial}{\partial \xi} u_{\xi}^{n+1}\right\}\right\} \\
& +\eta \mathbb{F}^{-1}\left\{k^{y-1} \mathbb{F}\left\{\rho^{n+1}\right\}\right\} .
\end{aligned}
$$

Here the temporal derivative of the acoustic density in the absorption term has been replaced using the linearized mass conservation equation $\partial \rho / \partial t=-\rho_{0} \nabla \cdot \mathbf{u}$ analogous to the first-order substitutions made in Sec. III B.

The discrete equations in Eq. (11) are iteratively solved using a time step based on the Courant-Friedrichs-Lewy (CFL) number, where $\Delta t=\mathrm{CFL} \Delta x / c_{\max }$. A CFL number of 0.3 typically provides a good balance between accuracy and computational speed for weakly heterogeneous media. ${ }^{6}$ At each time step, a mass or force source can be included by adding the source values to the appropriate grid points within the computational domain. Similarly, the output from the simulation can be obtained by recording the acoustic variables at each time step at particular grid points. For regularly spaced Cartesian grids, the gradients can be computed efficiently using the fast Fourier transform (FFT). For the simulations presented here, a split-field PML was implemented to prevent waves from wrapping around the domain. The grids were also spatially and temporally staggered to improve accuracy. ${ }^{6}$ The discrete equations were implemented in $\mathrm{C}++$ as an extension to the open source K-WAVE acoustics toolbox for MATLAB (Mathworks, Natick, MA). ${ }^{40}$ A description of the computer code is given in the Appendix.

\section{NUMERICAL ACCURACY}

\section{A. Accuracy of the $k$-space operator for wave propagation in heterogeneous media}

In the limit of linear wave propagation in a lossless and homogeneous medium, the $k$-space pseudospectral discretization of the three coupled governing equations is exact, and the algorithm is unconditionally stable. Although the finite difference time step still introduces unwanted numerical dispersion (or phase error) as expected, this is corrected by the $k$-space operator $\kappa$ that appears in Eqs. (11a) and (11c). Provided the scalar sound speed used in the $k$-space operator $c_{\text {ref }}$ is chosen to match the sound speed in the medium $c_{0}$, this correction is exact. However, in the case of heterogeneous media, there will necessarily be regions of the medium where the local value of $c_{0}$ does not match the value of $c_{\text {ref }}$. Consequently, the phase correction provided by $\kappa$ will no longer be exact, and unwanted numerical dispersion will still be introduced into the solution.

To illustrate the effect of a mismatched $c_{\text {ref }}$ and $c_{0}$, the phase error as a function of $c_{\text {ref }}$ for a homogeneous medium with $c_{0}=1500 \mathrm{~m} / \mathrm{s}$ is shown in Fig. 1. This error corresponds to the numerical dispersion (as a percentage of $c_{0}$ ) in the propagation of a plane wave after 50 wavelengths using four grid points per wavelength and a CFL parameter of 0.3 . When $c_{\text {ref }}=c_{0}$, the phase correction provided by the $k$-space operator is exact and there is no phase error. When $c_{\text {ref }} \approx c_{0}$, the correction is no longer exact. However, the use of the $k$-space operator still provides a significant reduction in the phase error as compared to that introduced by a finite

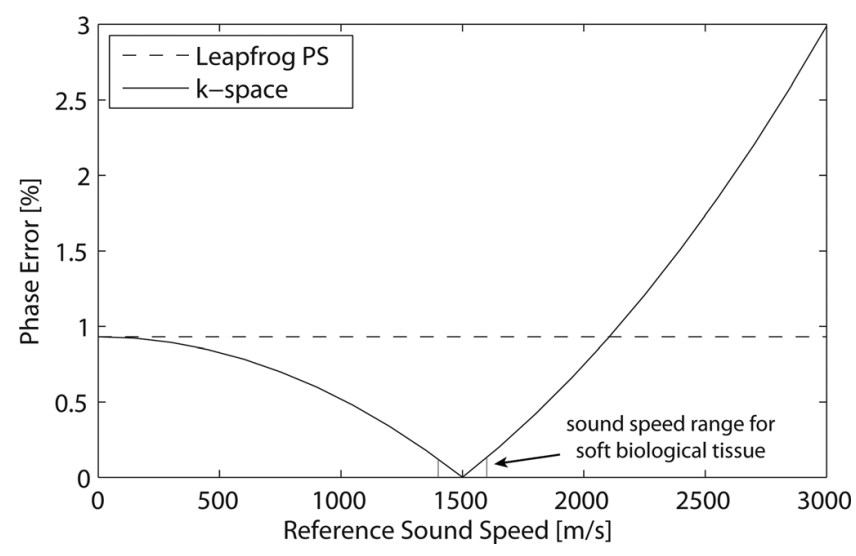

FIG. 1. Phase error in the propagation of a plane wave after 50 wavelengths against the reference sound speed $c_{\text {ref }}$ used in the $k$-space operator $\kappa$ for $c_{0}=1500 \mathrm{~m} / \mathrm{s}$. 
difference time step in the absence of this correction (dashed line shown in Fig. 1). Consequently, for soft biological tissue where the medium parameters are only weakly heterogeneous, the $k$-space method remains an apposite numerical technique.

It is useful to note, in the limit as $c_{\text {ref }}$ approaches $0, \kappa$ approaches 1 and thus the $k$-space operator has no effect (see Fig. 1). Conversely, for $c_{\text {ref }} \gg c_{0}$, this operator over compensates for the actual phase error introduced by the finite difference time step and thus increases the total phase error that is observed. Consequently, for modeling wave propagation in media with strong sound speed contrasts, care should be taken to select an appropriate reference sound speed, in addition to ensuring the appropriate stability constraints are met. ${ }^{6}$ In the case of strongly heterogeneous media, if the maximum phase error introduced by the finite difference time step is still unacceptable after the $k$-space correction, a higher order finite difference scheme could alternatively be used for the temporal discretization.

\section{B. Accuracy of the Fourier-collocation spectral method for heterogeneous media}

The Fourier-collocation spectral method used for the computation of the spatial derivatives in Eqs. (11) decomposes the pressure and velocity fields into a discrete Fourier series with a finite number of coefficients. This decomposition is accurate for periodic fields that vary sufficiently smoothly throughout the computational domain such that they can be accurately represented using the band-limited set of supported frequencies (there is an explicit and well understood relationship between the smoothness of a function and the rate of decay of its Fourier coefficients ${ }^{41}$ ). However, this is not the case when there are sharp gradients in the acoustic fields. These can occur when the field variables are multiplied by heterogeneous medium parameters, for example, in Eq. (11f). In this case, the band-limited Fourier representation of the acoustic fields will exhibit oscillations (analogous to Gibbs' phenomenon) and will no longer provide an accurate representation of the discontinuities as they appear in the continuous domain.

To investigate the error introduced when the medium parameters are heterogeneous, the accuracy of the transmission and reflection coefficients for a plane wave traveling through a step change in the ambient density and sound speed was examined. The resulting coefficients for a $10 \%$ change in the material parameters are shown in Fig. 2(a) along with their theoretical values (shown as straight solid lines). At the Nyquist sampling limit (two grid points per wavelength), there is a large error in the calculated coefficients. However, this reduces quickly as the number of grid points is increased. The corresponding results using both first-order and fourth-order accurate finite difference schemes (including staggered grids) for the computation of the spatial derivative are shown in Fig. 2(b), with the relative errors in the transmission coefficient shown in Fig. 2(c). To achieve an error in the transmission coefficient of less than $1 \%$, the Fourier-collocation spectral method requires only three grid points per wavelength, the fourth-order accurate (a)

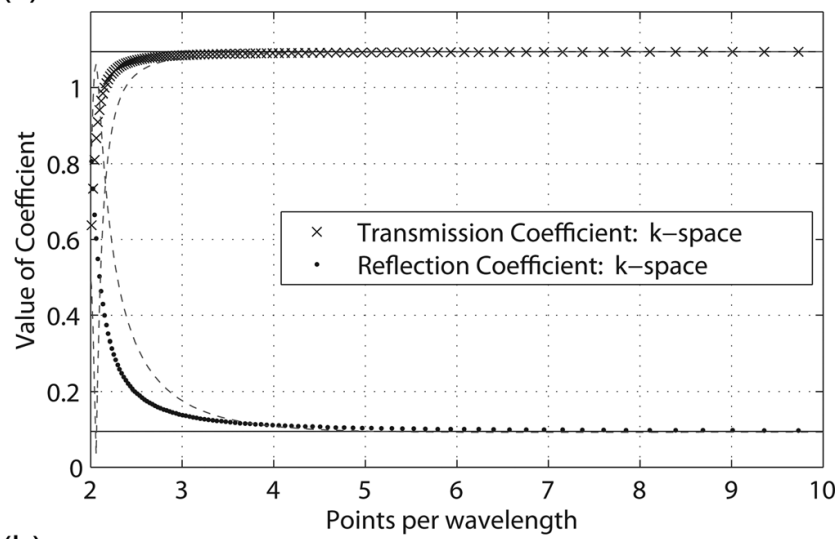

(b)

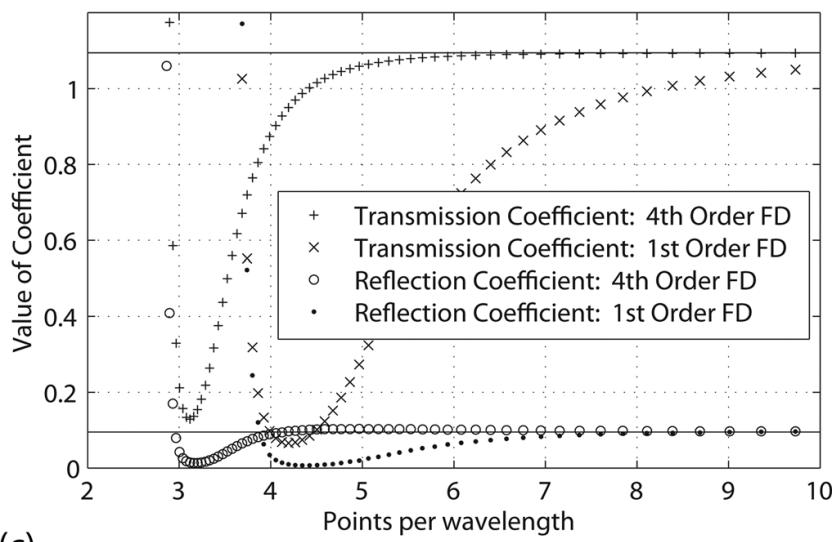

(c)

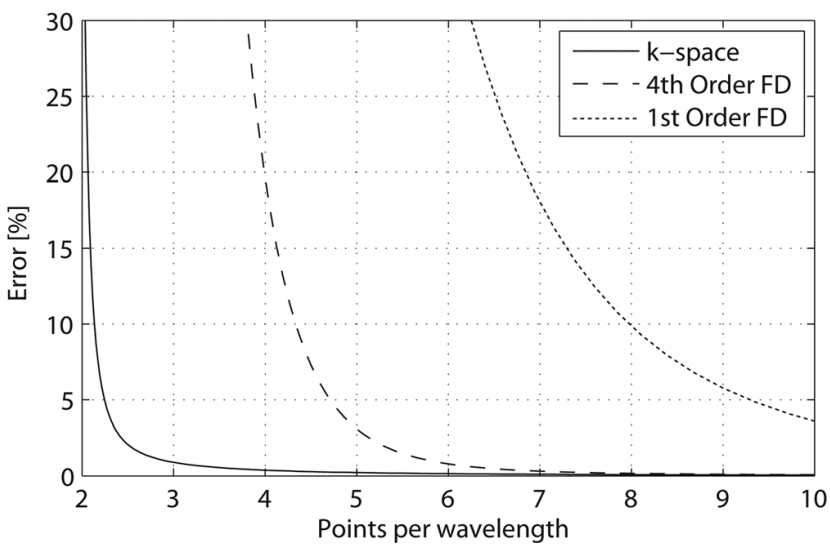

FIG. 2. (a) Transmission and reflection coefficients computed using the $k$ space model for a $10 \%$ step change in sound speed and density against the number of grid points per wavelength. The dashed lines show the results without the use of the staggered grid. (b) Analogous results when the spatial gradients are computed using a first-order and fourth-order accurate finite difference scheme. (c) Corresponding error in the transmission coefficient with the number of grid points used per wavelength.

finite difference scheme requires six points per wavelength, and the first-order accurate finite difference scheme requires 14. Similar results are observed for the reflection coefficient, although the overall errors are increased due to the smaller value of the coefficient (the error in the reflection coefficient for the Fourier-collocation and fourth-order finite difference schemes are approximately the same after six grid points per wavelength). Overall, for three-dimensional simulations, using a fourth-order accurate finite difference scheme 
requires around a 10 -fold increase in the total number of grid points to achieve the same level of accuracy, while using a first-order scheme requires a 100-fold increase. This corresponds to a significant increase in the computer memory required for a given simulation. For a given CFL, this also increases the number of time steps required. Similar results have previously been shown for scattering problems in two and three dimensions. ${ }^{6,42}$

\section{Nonlinear propagation in media with thermoviscous absorption}

To investigate the accuracy of the $k$-space model for simulating nonlinear wave propagation in absorbing media, the propagation of a monochromatic plane wave was compared to the analytical solution of Burgers equation derived by Mendousse. ${ }^{7,43}$ This solution accounts for thermoviscous absorption (which is proportional to frequency squared) for a source condition equal to $p=p_{0} \sin \left(2 \pi f_{0} t\right)$. It is convenient to describe the contribution of nonlinearity to the shape of the waveform using the non-dimensional shock parameter $\sigma$. For a monochromatic plane wave this is defined as

$$
\sigma=\frac{\beta p_{0} 2 \pi f_{0} x}{\rho_{0} c_{0}^{3}}
$$

where $x$ is the distance between the observation point and the source. A comparison between the $k$-space model and Mendousse's solution is shown in Fig. 3(a) for $\sigma=1$, where $\beta=4.8, p_{0}=5 \mathrm{MPa}, f_{0}=1 \mathrm{MHz}, \rho_{0}=1000 \mathrm{~kg} / \mathrm{m}^{3}$, $c_{0}=1500 \mathrm{~m} / \mathrm{s}$, and $\alpha_{0}=0.25 \mathrm{~dB} \mathrm{MHz}^{-2} \mathrm{~cm}^{-1}$. The $k$-space discretization used 30 grid points per wavelength at $f_{0}$ (supporting at most 15 harmonics) and a CFL number of 0.3 . The amplitudes of the first ten harmonics are shown in Fig. 3(b). There is a close agreement between the two models illustrating that acoustic absorption and cumulative nonlinear effects are correctly encapsulated. The corresponding waveform calculated using Eq. (11) with $\kappa=1$ (equivalent to a leapfrog pseudospectral model) is also shown. In this case, additional phase error is noticeable near the maximum and minimum of the waveform. The corresponding errors as a function of the CFL number are shown in Fig. 3(b), where the least squares error metric is defined as

$$
\operatorname{error}[\%]=100 \frac{\sum\left(p_{k-\text { space }}(t)-p_{\text {mendousse }}(t)\right)^{2}}{\sum\left(p_{\text {mendousse }}(t)\right)^{2}} \text {. }
$$

It is evident from this example that the $k$-space operator still significantly improves the accuracy of the solution, even when the governing equations include additional nonlinearity and absorption terms.

A second comparison is shown in Fig. 4 for a varying shock parameter again using 30 grid points per wavelength at $f_{0}$ and a CFL number of 0.3 . For low values of the shock parameter, the range of spatial wave numbers supported by the computational grid is sufficient to accurately represent the waveform. Consequently, the least squares error is small. As the shock parameter is increased, harmonics with wavelengths smaller than that supported by the grid spacing are (a)

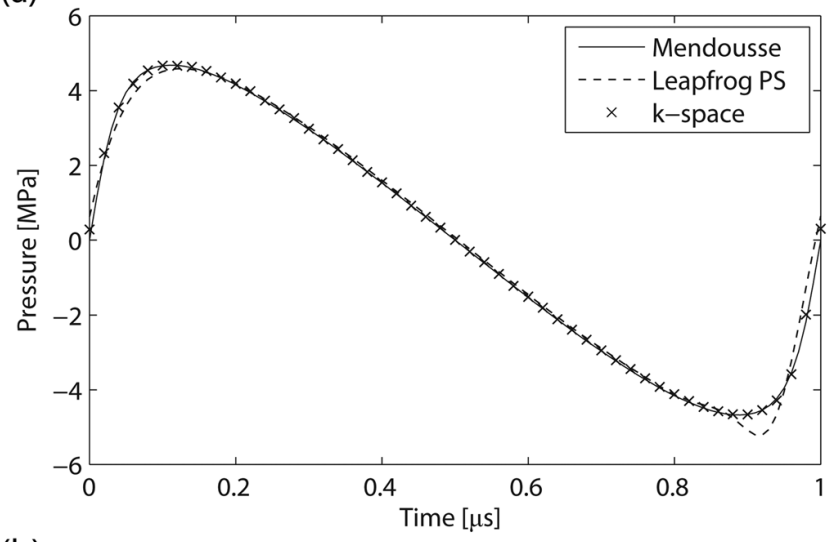

(b)

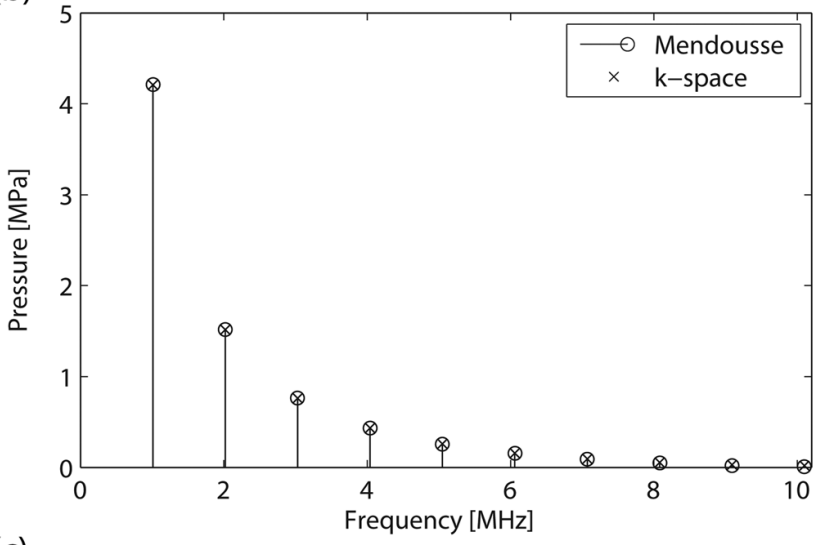

(c)

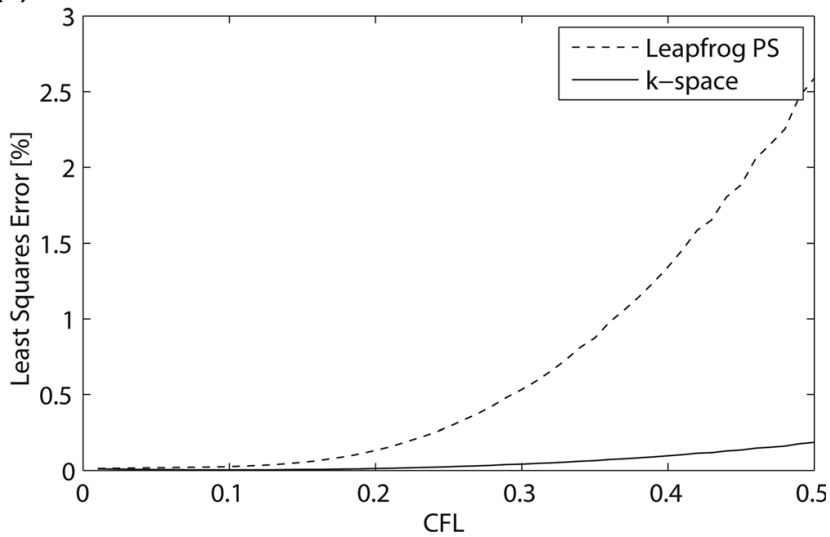

FIG. 3. (a) Comparison between Mendousse's analytical solution for the nonlinear propagation of a plane wave in a lossy medium and the nonlinear $k$-space model for a shock parameter of $\sigma=1$. (b) Corresponding amplitudes of the first 10 harmonics. (c) Change in the least squares error with the size of the time step defined by the Courant-Friedrichs-Lewy (CFL) number.

generated. This creates an aliasing effect known as spectral blocking in which wavenumbers higher than the Nyquist limit are aliased to wavenumbers supported by the computational grid. ${ }^{41}$ This effect is visible in Fig. 4(b) for $\sigma=3$. In this case, the amplitudes of the generated harmonics no longer decay, and the energy at $15 \mathrm{MHz}$ is erroneously greater than at $14 \mathrm{MHz}$ due to aliasing.

For a given grid size, there are several possible strategies to overcome spectral blocking (see Ref. 41, and references therein). For systems with a quadratic nonlinearity, 
(a)

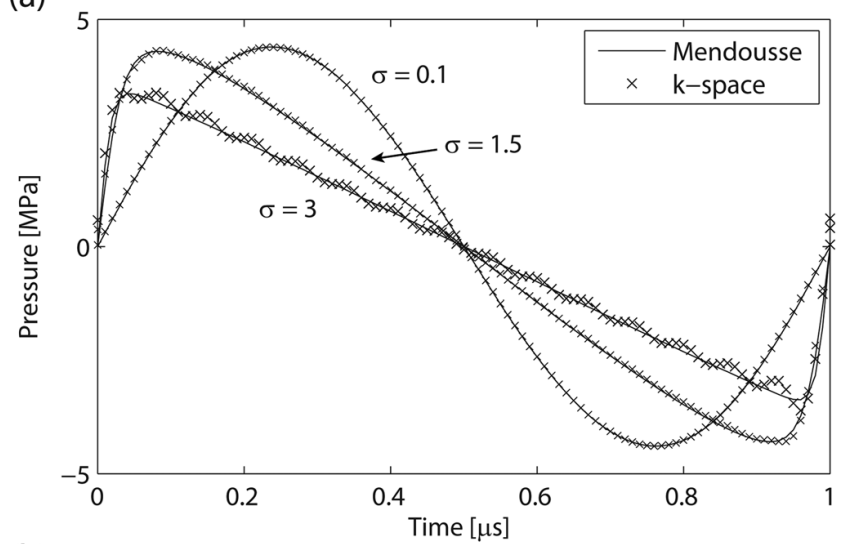

(b)

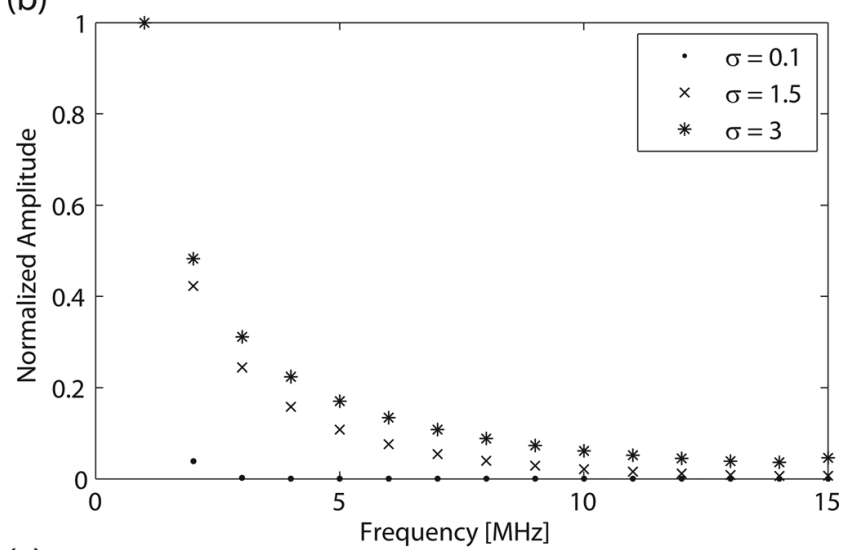

(c)

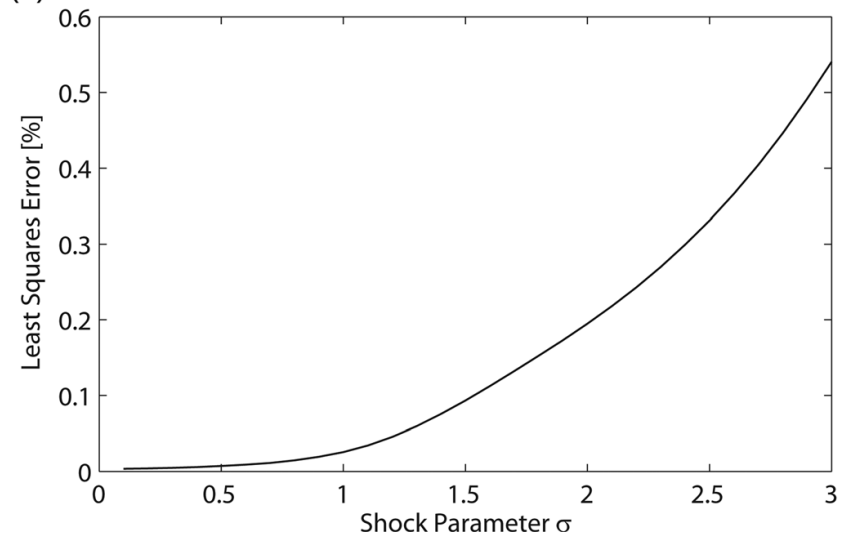

FIG. 4. (a) Comparison between Mendousse's analytical solution and the nonlinear $k$-space model for a varying shock parameter using 30 grid points per wavelength at $1 \mathrm{MHz}$. (b) Corresponding harmonic amplitudes calculated by the $k$-space model. (c) Change in the least squares error as a function of the shock parameter.

aliasing can be counteracted by increasing the grid size in each spatial dimension by a factor of $1 / 3$ and then filtering out the additional wavenumbers after each time step. For convective nonlinearity in the case of incompressible flow, aliasing errors can also be reduced by using the skewsymmetric form of the convective term. However, while these strategies can help minimize aliasing errors, the overall solution will still be inaccurate if there is significant energy at wavenumbers not supported by the computational grid. ${ }^{41}$ This is not a problem specific to spectral methods, rather, it is applicable regardless of the chosen numerical method. In this case, if the accurate calculation of the total acoustic pressure field is the desired outcome, the best strategy is to monitor the computed wavenumbers for spectral blocking, and increase the number of grid points used in the simulation if significant aliasing is observed.

More generally, the accuracy of the $k$-space model is dependent on several parameters. First, the number of grid points used per wavelength will control whether the computational grid can support the propagation of the generated harmonics. In turn, the rate at which these harmonics are produced will depend on the shock parameter (for example, the source strength and the coefficient of nonlinearity), while the rate at which they are absorbed will depend on the power law absorption parameters. Finally, the CFL number will control the amount of unwanted numerical dispersion introduced by the finite difference time step, as well as the accuracy with which the nonlinearity and absorption terms in the pressure-density relation are computed.

\section{Linear propagation in media with power law absorption}

To investigate the accuracy of the power law absorption term for general absorption parameters, the pressure field produced by a rectangular piston in an absorbing medium was compared to the fast near-field method as implemented in the FOCUS toolbox. ${ }^{44}$ This approach is comparable to evaluating the Rayleigh-Sommerfeld integral, but converges more rapidly by using an equivalent integral expression that removes numerical singularities. A comparison between the two models for a $6 \mathrm{~mm} \times 6 \mathrm{~mm}$ rectangular piston driven by a $3 \mathrm{MHz}$ sinusoid is shown in Fig. 5. To capture the rapid field variations close to the piston surface, the $k$-space model used four grid points per wavelength, with an overall computational grid size of $384 \times 128 \times 128$ grid points. The medium parameters were set to $c_{0}=1510 \mathrm{~m} / \mathrm{s}, \rho_{0}=1020 \mathrm{~kg} / \mathrm{m}^{3}$, and $\alpha_{0}=0.25 \mathrm{~dB} \mathrm{MHz}^{-1} \mathrm{~cm}^{-1}$. The beam pattern for the $k$-space model was produced by taking the maximum steady state pressure at each grid point. There is excellent agreement between the two models which confirms that absorption and near-field effects are correctly modeled.

\section{E. Simulation of nonlinear ultrasound beam patterns}

To illustrate the applicability of the developed nonlinear $k$-space model to ultrasound simulation more generally, the beam pattern produced by an Ultrasonix L9-4/38 linear array probe in a heterogeneous medium was investigated. This probe has 128 rectangular transducer elements with an element pitch of $304.8 \mu \mathrm{m}$, an elevation height of $6 \mathrm{~mm}$, and an elevation focus of $19 \mathrm{~mm}$. The beam pattern was computed in three dimensions using 32 active elements and an electronic focus of $15 \mathrm{~mm}$. The computational grid used including the PML was $1024 \times 512 \times 512$ grid points with a grid point spacing of $30.5 \mu \mathrm{m}$. This corresponds to a maximum frequency of $25.2 \mathrm{MHz}$ at two grid points per wavelength, or $16.8 \mathrm{MHz}$ at three grid points per wavelength (giving a computational domain size of $340 \times 170 \times 170$ wavelengths at the maximum frequency). The transducer was driven by a five cycle tone burst with a center frequency of $5 \mathrm{MHz}$ and an equivalent source pressure of $0.25 \mathrm{MPa}$ per grid node of 
(a)
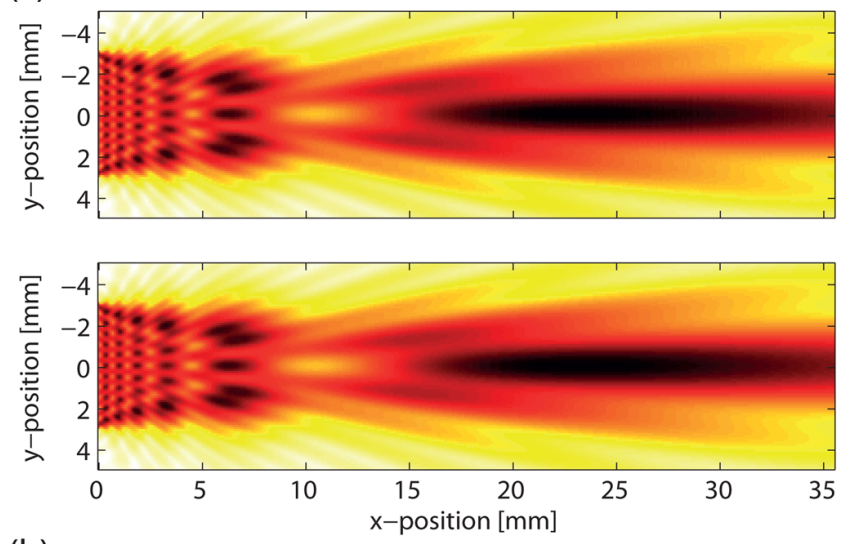

(b)

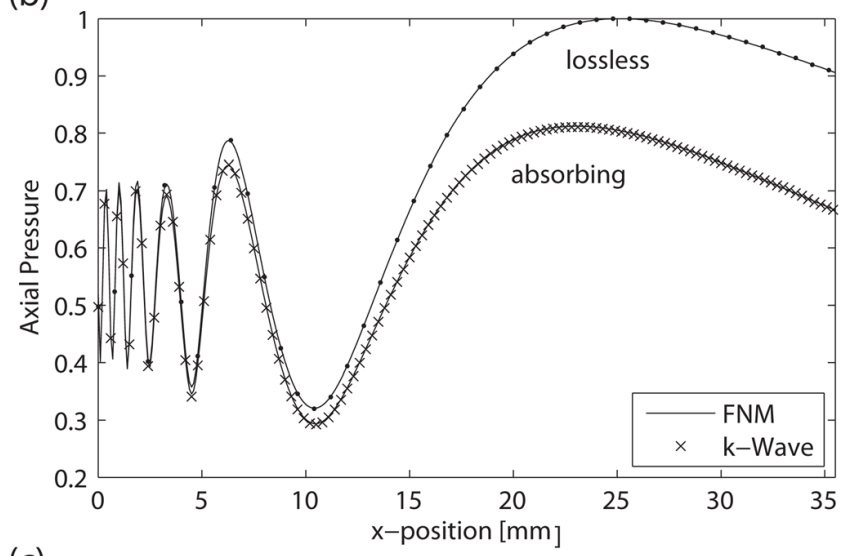

(c)

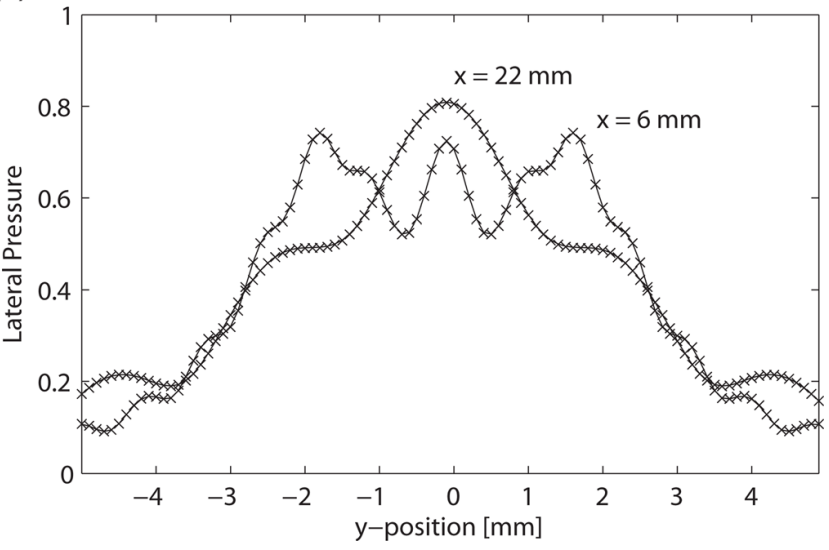

FIG. 5. (Color online) (a) Azimuth plane beam pattern generated by a rectangular piston in an homogeneous absorbing medium using the $k$-space method (top panel) and the fast near-field method (bottom panel). (b) Comparison of the pressure maximum along the transducer axis. (c) Comparison of the lateral pressure at $x=6 \mathrm{~mm}$ and $x=22 \mathrm{~mm}$.

each transducer element. The input signal was assigned to the $\mathrm{x}$-direction particle velocity (rather than the pressure) to mimic the directionality of the physical piezoelectric transducer elements. Each element was represented by 9 grid points in the azimuth direction (with a kerf width of 1 grid point) and 196 grid points in the elevation direction. The beamforming delays were calculated geometrically, and the elevation focus was modeled by applying additional beamforming delays across the grid points in the elevation direction. The CFL number was set to 0.3 giving a time step of $6 \mathrm{~ns}$, and the simulation was run for 4000 time steps.
For the homogeneous medium, the properties were set to those of breast tissue, where $c_{0}=1510 \mathrm{~m} / \mathrm{s}, \rho_{0}=1020$ $\mathrm{kg} / \mathrm{m}^{3}, \quad B / A=9.63, \quad \alpha_{0}=0.75 \mathrm{~dB} \mathrm{MHz}^{-y} \mathrm{~cm}^{-1}, \quad$ and $y=1.5{ }^{1}$ For the heterogeneous medium, the sound speed and density maps were derived from a CT scan of a human neck. To simulate small scale heterogeneities, the values of the sound speed and density at each grid point were scaled by a Gaussian random variable with a mean of 1 and a standard deviation of 0.05 . Each simulation took $7.5 \mathrm{~h}$ to run and used 27 GB of memory (using the Tyan server in the 48 GB configuration; details are given in the Appendix). The generated azimuth and elevation plane beam patterns are shown in Fig. 6. The total beam patterns were produced by taking the maximum value of the pressure recorded at each grid point, while the beam patterns at the second harmonic correspond to the relative spectral amplitudes at this frequency. When the medium is heterogeneous, the variations in the medium parameters alter both the shape and the position of the beam focus. By recording the acoustic signals reflected back to the active transducer elements, it is straightforward to extend the simulations to form B-mode ultrasound images. ${ }^{36}$

\section{F. Comparison with other full-wave nonlinear models}

The computational complexity of solving general nonlinear equations means only a limited number of threedimensional full-wave models have previously been reported in the literature. Pinton et al., ${ }^{2}$ recently presented a solution to the heterogeneous Westervelt equation with a relaxation absorption term using a second-order-in-time, fourth-orderin-space finite difference method. Simulations using computational grid sizes on the order of $800 \times 800 \times 800$ grid points were run on a distributed cluster with run times on the order of $32 \mathrm{~h}$. Comparatively, the $k$-space pseudospectral method reduces the number of grid points and time steps required for the same level of accuracy.

Verweij and Huijssen ${ }^{3,12}$ also recently presented an iterative method to solve the linear homogeneous wave equation with absorption, nonlinearity, and heterogeneity included as contrast source terms. This approach allows both the spatial and temporal fields to be sampled at the Nyquist limit (equivalent to a CFL number of 1). It also provides a mechanism for high frequency harmonics not supported by the computational grid to be removed via spatiotemporal filtering. However, a significant disadvantage is that the complete time history of the field data must be stored to allow the evaluation of the required convolutions. This considerably increases memory requirements. For the example discussed in Sec. V E, even after accounting for a reduction in the total number of grid points by a factor of 8 (assuming the $k$-space model requires a conservative four grid points per wavelength at the maximum frequency of interest) and using a CFL number of 1 , the storage of the time history of one field variable in single precision still requires $75 \mathrm{~GB}$ of memory.

\section{SUMMARY}

A set of coupled first-order equations valid for modeling nonlinear wave propagation in heterogeneous media with power law absorption is derived. The additional terms 

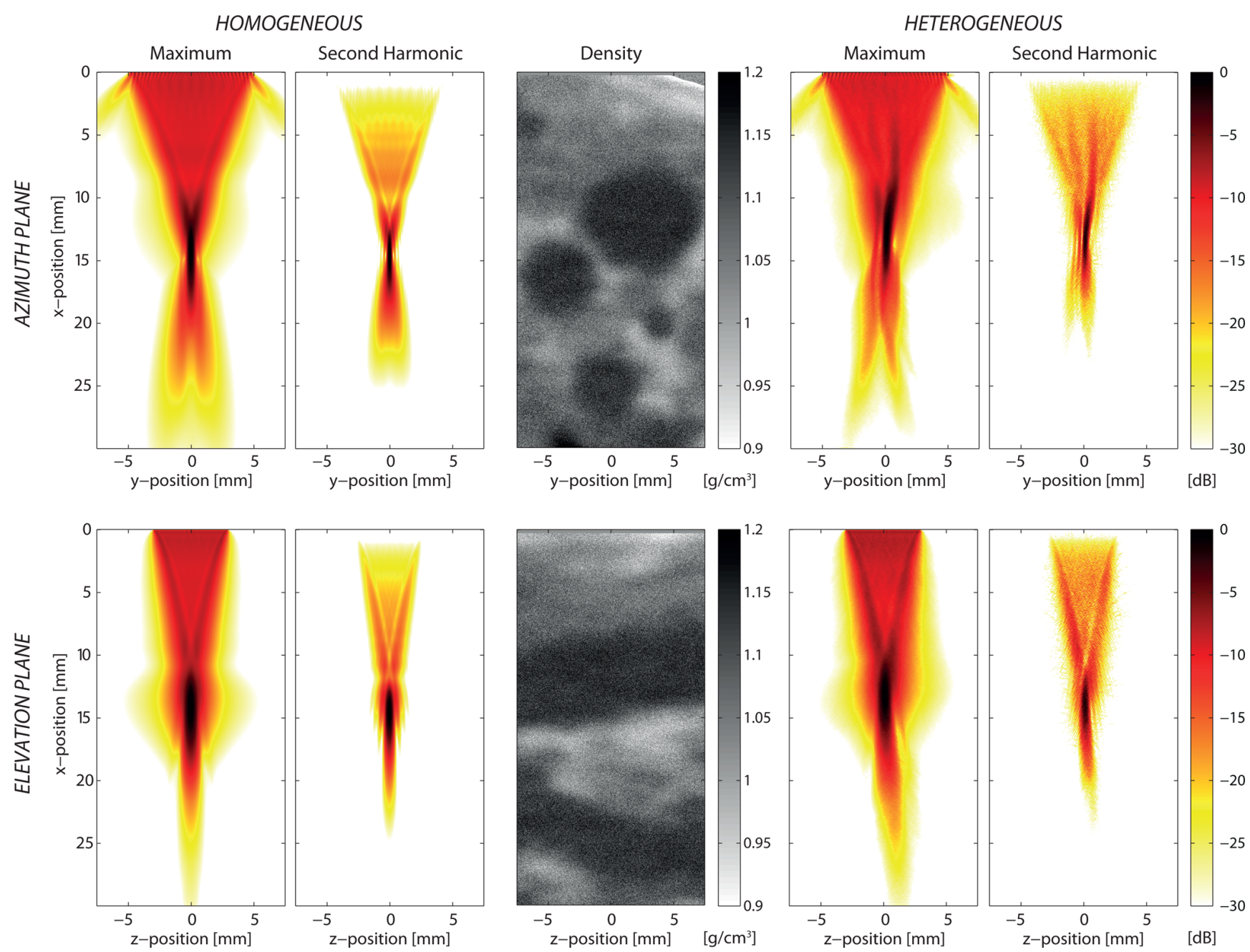

FIG. 6. (Color online) Normalized azimuth and elevation plane beam patterns generated by a clinical linear array ultrasound transducer for a homogeneous medium and a heterogeneous medium using a density map derived from a CT scan.

accounting for cumulative nonlinear effects and power law absorption are expressed as spatial gradients which makes them efficient to numerically encode. The derived governing equations are then discretized using the $k$-space pseudospectral method. The use of the $k$-space operator significantly reduces the phase error introduced by the finite difference time step, allowing larger time steps to be taken for the same level of accuracy. The use of the Fouriercollocation spectral method similarly improves the accuracy of the spatial gradient calculations which relaxes the requirement for dense computational grids compared to conventional finite difference methods. A number of numerical examples are given to illustrate the accuracy of the model. The utility of the nonlinear $k$-space model is then demonstrated via the three-dimensional simulation of the beam pattern from a clinical ultrasound transducer in both homogeneous and heterogeneous media. Compared to previous ultrasound models based on the KZK equation, the current model does not have any restrictions on the directionality or spatial variation of the sound waves. This facilitates arbitrary full-wave simulations of cumulative nonlinear effects in tissue-realistic media. The model is particularly relevant to the simulation of diagnostic and thera- peutic ultrasound fields in heterogeneous media, as well as the generation of full-wave harmonic ultrasound images.

\section{ACKNOWLEDGMENTS}

This work was supported in part by the Australian Research Council/Microsoft Linkage Project LP100100588 and the Engineering and Physical Sciences Research Council, UK. The authors would like to thank Anthony Harker, Mustafa Tumen, Nimalan Nandapalan, and Trond Varslot for useful discussion.

\section{APPENDIX: COMPUTER IMPLEMENTATION}

The discrete equations described in Sec. IV B were implemented in $\mathrm{C}++$ as an extension to the open source K-WAVE toolbox. ${ }^{40}$ The codes were optimized to run using a Tyan server (MiTAC, Taipei, Taiwan) with two six-core Intel Xeon X5650 processors. To maximize computational efficiency, several stages of code optimization were performed. ${ }^{45}$ First, the 3D FFTs were computed using the realto-complex FFT from the FFTW library. Compared to the complex-to-complex FFT, this reduced the compute time 


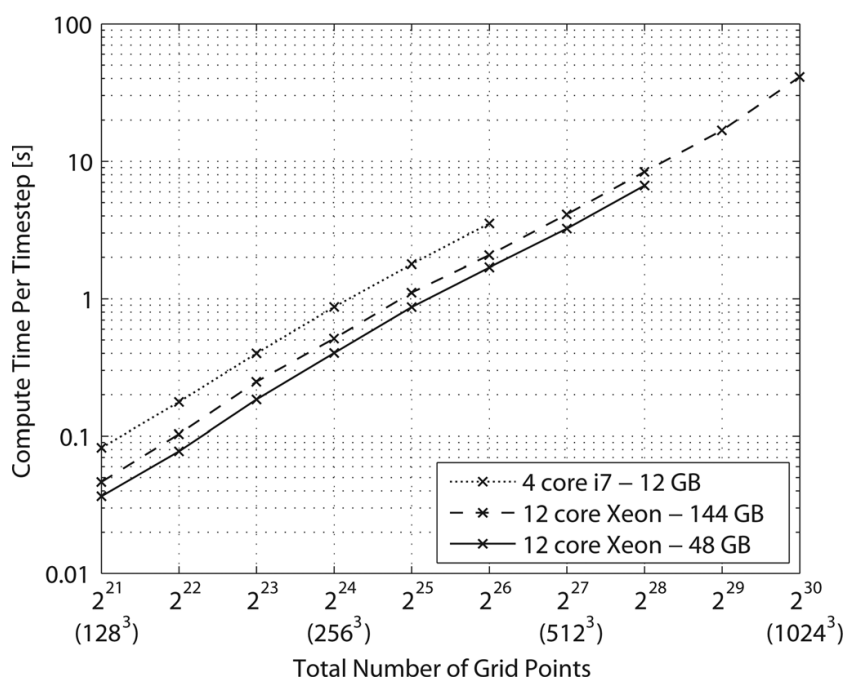

FIG. 7. Compute times per time step for the nonlinear $k$-space model for different $3 \mathrm{D}$ grid sizes. The time complexity is on the order of $\mathrm{O}(n \log n)$ where $n=N x \times N y \times N z$.

and memory associated with the FFT by nearly $50 \%$. Second, to save memory bandwidth, all operations were computed in single precision. Parameters such as the PML and wavenumber operators were stored as $1 \mathrm{D}$ vectors and virtually replicated to $3 \mathrm{D}$ as needed via indexing. Third, elementwise operations were parallelized using OPENMP and then optimized using streaming SIMD extensions (SSE). Multiple mathematical operations were applied to each grid point where possible to maximize temporal data locality. Finally, as the Tyan server is based on the non-uniform memory access architecture, policies were implemented to bind threads to cores and allocate memory to nearby memory locality domains. $^{45}$

The compute times per time step for a range of computational grid sizes are shown in Fig. 7. The three curves correspond to three different computer configurations: a desktop computer with a four-core Intel Core i7 950 processor and 12 GB of DDR3 RAM, and the Tyan server with either $144 \mathrm{~GB}(18 \times 8 \mathrm{~GB})$ or $48 \mathrm{~GB}(12 \times 4 \mathrm{~GB})$ of DDR3 RAM. The performance difference between the two memory configurations for the Tyan server is due to a reduction in the memory speed from $1333 \mathrm{MHz}$ to $1066 \mathrm{MHz}$ when the memory channels are fully populated. The memory usage for a given grid size can be estimated by

$$
\text { memory usage }[\mathrm{GB}] \approx \frac{21 N x N y N z+9 \frac{N x}{2} N y N z}{1024^{3} / 4},
$$

where $N x, N y$, and $N z$ are the grid sizes in the $x, y$, and $z$ directions. The first term accounts for 21 real matrices and the second term accounts for 3 real and 3 complex matrices in the spatial Fourier domain. The relatively large number of $3 \mathrm{D}$ matrices is required to store the heterogeneous material parameters, field values, and temporary results.

For a computational grid with $512 \times 512 \times 512$ grid points, the overall performance of the $k$-space model running on the Tyan server (in the 48 GB memory configuration) was approximately 17 GFLOPS. In this case, the maximum achievable performance was limited by the available memory bandwidth as many of the element-wise operations have limited or no data re-usage so benefit little from the availability of cache. For comparison, the LINPACK benchmark from the Intel Math Kernel Library (which is used to test the theoretical peak performance of Intel processors) had a performance of 118 GFLOPS, while the 3D real-to-complex FFT in isolation had a performance of 42 GFLOPS. For a representative simulation, around $60 \%$ of the total computation time is spent performing the forward and inverse FFT.

${ }^{1}$ T. L. Szabo, Diagnostic Ultrasound Imaging (Elsevier, Burlington, 2004), pp. 4-6.

${ }^{2}$ G. F. Pinton, J. Dahl, S. Rosenzweig, and G. E. Trahey, "A heterogeneous nonlinear attenuating full-wave model of ultrasound," IEEE Trans. Ultrason. Ferroelectr. Freq. Control 56, 474-488 (2009).

${ }^{3}$ J. Huijssen and M. D. Verweij, "An iterative method for the computation of nonlinear, wide-angle, pulsed acoustic fields of medical diagnostic transducers," J. Acoust. Soc. Am. 127, 33-44 (2010).

${ }^{4} \mathrm{~V}$. W. Sparrow and R. Raspet, "A numerical method for general finite amplitude wave propagation in two dimensions and its application to spark pulses," J. Acoust. Soc. Am. 90, 2683-2691 (1991).

${ }^{5} \mathrm{~J}$. A. Jensen, "A model for the propagation and scattering of ultrasound in tissue," J. Acoust. Soc. Am. 89, 182-190 (1991).

${ }^{6} \mathrm{M}$. Tabei, T. D. Mast, and R. C. Waag, "A k-space method for coupled first-order acoustic propagation equations," J. Acoust. Soc. Am. 111, 53-63 (2002).

${ }^{7}$ Nonlinear Acoustics, edited by M. F. Hamilton and D. T. Blackstock (Acoustical Society of America, Melville, 2008), pp. 1-455.

${ }^{8}$ G. Taraldsen, "A generalized Westervelt equation for nonlinear medical ultrasound," J. Acoust. Soc. Am. 109, 1329-1333 (2001).

${ }^{9} \mathrm{~F}$. Coulouvrat, "New equations for nonlinear acoustics in a low Mach number and weakly heterogeneous atmosphere," Wave Motion 49, 50-63 (2012)

${ }^{10}$ I. M. Hallaj, R. O. Cleveland, and K. Hynynen, "Simulations of the thermo-acoustic lens effect during focused ultrasound surgery," J. Acoust. Soc. Am. 109, 2245-2253 (2001).

${ }^{11} \mathrm{Y}$. Jing and R. O. Cleveland, "Modeling the propagation of nonlinear three-dimensional acoustic beams in inhomogeneous media," J. Acoust. Soc. Am. 122, 1352-1364 (2007).

${ }^{12} \mathrm{M}$. D. Verweij and J. Huijssen, "A filtered convolution method for the computation of acoustic wave fields in very large spatiotemporal domains," J. Acoust. Soc. Am. 125, 1868-1878 (2009).

${ }^{13} \mathrm{Y}$. Jing and G. T. Clement, "A k-space method for nonlinear wave propagation," arXiv:1105.2210.

${ }^{14}$ M. V. Averyanov, V. A. Khokhlova, O. A. Sapozhnikov, P. Blanc-Benon, and R. O. Cleveland, "Parabolic equation for nonlinear acoustic wave propagation in inhomogeneous moving media," Acoust. Phys. 52, 623-632 (2006).

${ }^{15}$ D. T. Blackstock, "Generalized Burgers equation for plane waves," J. Acoust. Soc. Am. 77, 2050-2053 (1985).

${ }^{16}$ T. L. Szabo, "Time domain nonlinear wave equations for lossy media," in Advances in Nonlinear Acoustics: Proceedings of the 13th International Symposium on Nonlinear Acoustics (World Scientific, Singapore, 1993), pp. 89-94.

${ }^{17}$ T. L. Szabo, "Time domain wave equations for lossy media obeying a frequency power law," J. Acoust. Soc. Am. 96, 491-500 (1994).

${ }^{18}$ J. Tavakkoli, D. Cathignol, R. Souchon, and O. A. Sapozhnikov, "Modeling of pulsed finite-amplitude focused sound beams in time domain,” J. Acoust. Soc. Am. 104, 2061-2072 (1998).

${ }^{19}$ J. Wojcik, "Conservation of energy and absorption in acoustic fields for linear and nonlinear propagation," J. Acoust. Soc. Am. 104, 2654 (1998).

${ }^{20} \mathrm{~W}$. Chen and S. Holm, "Fractional Laplacian time-space models for linear and nonlinear lossy media exhibiting arbitrary frequency power-law dependency," J. Acoust. Soc. Am. 115, 1424-1430 (2004).

${ }^{21}$ W. Chen and S. Holm, "Fractional Laplacian, Levy stable distribution, and time-space models for linear and nonlinear frequency-dependent lossy 
media," Technical Report, Research Report of Simula Research Laboratory (2002)

${ }^{22}$ B. E. Treeby and B. T. Cox, "Modeling power law absorption and dispersion for acoustic propagation using the fractional Laplacian," J. Acoust. Soc. Am. 127, 2741-2748 (2010).

${ }^{23}$ B. E. Treeby and B. T. Cox, "A k-space Greens function solution for acoustic initial value problems in homogeneous media with power law absorption," J. Acoust. Soc. Am. 129, 3652-3660 (2011).

${ }^{24}$ H. A. H. Jongen, J. M. Thijssen, M. van den Aarssen, and W. A. Verhoef, "A general model for the absorption of ultrasound by biological tissues and experimental verification," J. Acoust. Soc. Am. 79, 535-540 (1986).

${ }^{25}$ A. I. Nachman, J. F. Smith III, and R. C. Waag, "An equation for acoustic propagation in inhomogeneous media with relaxation losses," J. Acoust. Soc. Am. 88, 1584-1595 (1990).

${ }^{26}$ R. O. Cleveland, M. F. Hamilton, and D. T. Blackstock, "Time-domain modeling of finite-amplitude sound in relaxing fluids," J. Acoust. Soc. Am. 99, 3312-3318 (1996).

${ }^{27}$ S. P. Nasholm and S. Holm, "Linking multiple relaxation, power-law attenuation, and fractional wave equations," J. Acoust. Soc. Am. 130, 3038-3045 (2011).

${ }^{28}$ M. J. Lighthill, "Viscosity effects in sound waves of finite amplitudes," in Surveys in Mechanics, edited by G. K. Batchelor and R. M. Davies (Cambridge University Press, Cambridge, 1956), pp. 250-351.

${ }^{29}$ In a fluid model at equilibrium, a heterogeneous ambient density physically requires a body force to support it. In soft tissue this could be provided, for example, by stresses in the extracellular matrix. As the fluid is stationary in the ambient state, this body force must be matched by a gradient in the ambient pressure, where $\nabla p_{0}=\rho_{0}$ f. Because these terms exactly cancel, they are not included in the dynamic momentum equation given in Eq. (2a).

${ }^{30}$ A. D. Pierce, "Mathematical theory of wave propagation," in Handbook of Acoustics, edited by M. J. Crocker (Wiley, New York, 1998), pp. 21-37.

${ }^{31}$ F. Prieur and S. Holm, "Nonlinear acoustic wave equations with fractional loss operators," J. Acoust. Soc. Am. 130, 1125-1132 (2011).

${ }^{32}$ Y. Jing, D. Shen, and G. T. Clement, "Verification of the Westervelt equation for focused transducers," IEEE Trans. Ultrason. Ferroelectr. Freq. Control 58, 1097-1101 (2011).

${ }^{33}$ S. I. Aanonsen, T. Barkve, J. N. Tjotta, and S. Tjotta, "Distortion and harmonic generation in the nearfield of a finite amplitude sound beam," J. Acoust. Soc. Am. 75, 749-768 (1984).
${ }^{34}$ M. F. Hamilton and D. T. Blackstock, "On the linearity of the momentum equation for progressive plane waves of finite amplitude," J. Acoust. Soc. Am. 88, 2025-2026 (1990).

${ }^{35}$ Subtly, this means the acoustic density calculated by the discrete equations is not exactly equal to the true acoustic density as defined in the general conservation equations. However, because the acoustic density is not generally used for output, this difference does not affect the accuracy of the simulations.

${ }^{36} \mathrm{~B}$. E. Treeby, M. Tumen, and B. T. Cox, "Time domain simulation of harmonic ultrasound images and beam patterns in 3D using the k-space pseudospectral method," in Medical Image Computing and ComputerAssisted Intervention, Part I (Springer, Heidelberg, 2011), Vol. 6891, pp. 363-370.

${ }^{37}$ T. D. Mast, L. P. Souriau, D.-L. D. Liu, M. Tabei, A. I. Nachman, and R. C. Waag, "A k-space method for large-scale models of wave propagation in tissue," IEEE Trans. Ultrason. Ferroelectr. Freq. Control 48, 341-354 (2001).

${ }^{38}$ J.-P. Berenger, "Three-dimensional perfectly matched layer for the absorption of electromagnetic waves," J. Comput. Phys. 127, 363-379 (1996).

${ }^{39}$ B. T. Cox, S. Kara, S. R. Arridge, and P. C. Beard, "k-space propagation models for acoustically heterogeneous media: Application to biomedical photoacoustics," J. Acoust. Soc. Am. 121, 3453-3464 (2007).

${ }^{40}$ B. E. Treeby and B. T. Cox, "k-Wave: matLaB toolbox for the simulation and reconstruction of photoacoustic wave fields," J. Biomed. Opt. 15, 021314 (2010).

${ }^{41}$ J. P. Boyd, Chebyshev and Fourier Spectral Methods (Dover, Mineola, NY, 2001), pp. 202-221.

${ }^{42}$ Y. Jing, F. C. Meral, and G. T. Clement, "Time-reversal transcranial ultrasound beam focusing using a k-space method," Phys. Med. Biol. 57, 901-917 (2012).

${ }^{43}$ J. S. Mendousse, "Nonlinear dissipative distortion of progressive sound waves at moderate amplitudes," J. Acoust. Soc. Am. 25, 51-54 (1953).

${ }^{44}$ R. J. McGough, "Rapid calculations of time-harmonic nearfield pressures produced by rectangular pistons," J. Acoust. Soc. Am. 115, 1934-1941 (2004).

${ }^{45}$ J. Jaros, B. E. Treeby, and A. P. Rendell, "Use of multiple GPUs on shared memory multiprocessors for ultrasound propagation simulations," in 10th Australasian Symposium on Parallel and Distributed Computing, edited by J. Chen and R. Ranjan, ACS (2012), Vol. 127, pp. 43-52. 